\title{
The synchronicity of dynamic epistemic logic
}

\author{
Cédric Dégremont \\ Afdeling Kunstmatige Intelligentie \\ Rjiksuniversiteit Groningen \\ Bernoulliborg \\ Nijenborgh 9 \\ 9747 AG Groningen \\ The Netherlands
}

\author{
Benedikt Löwe \\ Institute for Logic, Language \\ and Computation \\ Universiteit van Amsterdam \\ Postbus 94242 \\ 1090 GE Amsterdam \\ The Netherlands
}

\author{
Andreas Witzel \\ NYU Bioinformatics Lab \\ Courant Institute for the \\ Mathematical Sciences \\ New York University \\ New York, NY 10003 \\ United States of America
}

\begin{abstract}
Van Benthem, Gerbrandy, Hoshi and Pacuit gave a natural translation of dynamic epistemic logic (DEL) into epistemic temporal logic (ETL) and proved a representation theorem, characterizing those ETL models that are translations of some DEL protocol; among the characterizing properties we also find synchronicity. In this paper, we argue that synchronicity is not an inherent property of DEL, but rather of the translation that van Benthem et al. used. We provide a different translation that produces asynchronous ETL models and discuss a minimal temporal extension of DEL that removes the ambiguities between the possible translations. This allows us a first attempt of an assessment which of the epistemic-temporal properties are intrinsic to DEL and which are properties of the translation.
\end{abstract}

\section{Introduction}

Dynamic epistemic logic (DEL) (Baltag et al., 1998), while being about changes in epistemic status over time, is not a temporal logic and cannot itself express temporal aspects. In order to model the temporal aspects of epistemic and doxastic change represented in dynamic epistemic logic, we need to embed DEL into some epistemic temporal system such as ETL Parikh and Ramanujam, 2003) or Interpreted Systems (Fagin et al., 1995). ETL is a close cognate to DEL in the sense that its also events do not include a notion of agency or turns, as opposed to some other multi-agent epistemic-temporal logics such as ATEL van der Hoek and Wooldridge, 2003).

Representation theorems linking DEL and ETL were proposed for the first time in (van Benthem, 2001). Recently, van Benthem, Gerbrandy, Hoshi, and Pacuit
2009 gave a natural model-based translation from the framework of DEL into ETL: for each DEL protocol, they produce an ETL forest generated by sequentially updating an epistemic model using the DEL product update. They then showed that the temporal translation of DEL thus obtained always produces ETL models satisfying a number of properties, including synchronicity and perfect recall. We shall refer to this result (precisely given as Theorem 6) by "the vBGHP representation".

The vBGHP representation has been interpreted to imply that DEL can inherently only model agents that are synchronous ${ }^{1}$ Our aim here is to stress that this is an oversimplification of the mentioned result; in fact, synchronicity is a property of the described translation, not a property of the logic. Other translations of DEL into ETL might very well exhibit other features.

Our aim is to clarify the meaning of the vBGHP representation, with the long-term goal to identify those properties that do not depend on the choice of embedding of DEL and ETL and those which do.

The paper is organized as follows: After giving the general framework $(\S 2$ and discussing the concept of perfect recall $(\S 3)$, we propose an alternative way of embedding DEL into ETL, exhibiting a natural example of an asynchronous ETL model that arguably represents faithfully the dynamic component of DEL (Example 13, and finally state a representation theorem for our embedding $(\S 4$. Comparing the properties characterizing asynchronously DEL generated forests (Theorem 16) to the properties used in the vBGHP representation (Corollary 9), we discuss which properties might be regarded as core DEL properties $(\$ 5)$.

Finally, we then generalize the two interpretations by introducing clock tick functions which allows to cover a whole range, having the two given constructions as

\footnotetext{
${ }^{1}$ E.g., (Renne et al. 2009, p. 1): "[van Benthem et al.] showed that standard Dynamic Epistemic Logic necessarily satisfies synchronicity".
} 
extremal special cases $(\S 6$. In $\S 7$, we note that the cases of asynchronicity are rather limited if we restrict our attention to S5 and sketch some avenues for future research, including an investigation of sub-S5 settings.

\section{Related Work.}

Our work fits in the research tradition at the interface between DEL and ETL which is, e.g., studied in (Sack, 2007) and (Hoshi, 2009) where the reader can find logics designed at this interface, merging the dynamic and the temporal paradigms. More results in this direction can be found in (Wang et al. 2010) and its unpublished extended version. The vBGHP representation (the main motivation of the present paper) is published in (van Benthem et al. 2009) together with other results about the merging of the dynamic and the temporal framework. A combination of temporal logic and dynamic epistemic logic can be found in (Renne et al., 2009).

In this paper, our interest is not so much the merging of the frameworks and the design of logics that have both dynamic and temporal aspects, but rather the study of the translation between the dynamic and the temporal framework. In this study, we encounter natural temporal properties such as synchronicity and perfect recall. These properties have been studied by other authors. E.g., Renne et al. (2009) extend basic DEL models and protocols to account for nonsynchronous scenarios and Isaac and Hoshi (to appear) discuss transformations of asynchronous models into synchronous models (thus resolving the diachronic uncertainty).

In our investigation of synchronicity of the translation used in the vBGHP representation, we notice that the notion of perfect recall used in the theorem in a sense presupposes synchronicity. This lead to a separate study of different notions of perfect recall in (Witzel, 2011). We shall use its analysis in our $\S 3$, and consider it a sibling to the present paper.

Finally, in $\S 7$, we discuss possible directions of future work such as weakening the S5 assumptions or adding more structure to the models. Papers relating to the research directions are listed and discussed in $\S 7$.

\section{Basic definitions and the vBGHP representation}

\subsection{DEL and ETL models and their properties}

In the following, we shall deal with various types of relational structures, building on the same sets of propositional variables and agents that we denote by PROP and $N$, respectively. Modal languages that can be interpreted on such structures could have three types of modalities: epistemic, temporal and action modalities.

An (S5) epistemic model $\mathcal{M}=\left(W,\left(\sim_{i}\right)_{i \in N}, V\right)$ consists of a nonempty set $W$ of worlds, an equivalence relation $\sim_{i}$ for every agent, and a valuation function $V:$ PROP $\rightarrow \wp(W)$. We also write $|\mathcal{M}|$ for $W$. A frame is a model without valuation function. Event models are triples $\mathcal{E}=\left\langle E,\left(\sim_{i}^{\mathcal{E}}\right)_{i \in N}\right.$, pre $\rangle$, where $E \neq \emptyset$ is a set of events, for each agent $i \in N, \sim_{i}^{\mathcal{E}}$ is an equivalence relation on $E$, and pre $: E \rightarrow \mathcal{L}$ is a precondition function mapping events into some epistemic language $\mathcal{L}$. As usual, a pointed event model is an event model with one distinguished element from $E$.

We shall not give an introduction to dynamic epistemic logic here, but rather refer the reader to the textbook (van Ditmarsch et al., 2007). The crucial operation for DEL is that of the product update:

Definition 1 (Product Update). The product update of an epistemic model $\mathcal{M}=\left\langle W,\left(\sim_{i}\right)_{i \in N}, V\right\rangle$ with an event model $\mathcal{E}=\left\langle E,\left(\sim_{i}^{\mathcal{E}}\right)_{i \in N}\right.$, pre $\rangle$ is the model $\mathcal{M} \otimes \mathcal{E}$ whose states are the pairs $(w, e)$ such that $w$ satisfies the precondition of the event $e$ and whose epistemic relations are defined as:

$$
(w, e) \sim_{i}^{\prime}\left(w^{\prime}, e^{\prime}\right) \text { iff } e \sim_{i}^{\mathcal{E}} e^{\prime}, w \sim_{i} w^{\prime}
$$

and whose valuation is defined by

$$
(w, e) \in V^{\prime}(p) \text { iff } w \in V(p), \text { for all } p \in \text { PROP } .
$$

We turn to structures giving a temporal perspective on information change: an (S5) epistemic temporal model or (S5) ETL model $\mathcal{H}$ is a tuple $\left\langle\Sigma, H,\left(\sim_{i}\right)_{i \in N}, V\right\rangle$ with $\Sigma$ a finite set of events, and $H \subseteq \Sigma^{*}$ closed under non-empty prefixes (i.e., is a forest of events). For each $i \in N, \sim_{i}$ is an equivalence relation on $H$, and there is a valuation $V:$ PROP $\rightarrow \wp(H)$.

We introduce some notation for ETL models that we shall use later. In the following, we fix an ETL model $\mathcal{H}=\left\langle\Sigma, H,\left(\sim_{i}\right)_{i \in N}, V\right\rangle$.

For any sequence $\sigma$, let $\sigma[n]$ be the $n$th element of $\sigma$. We write $h \leq h^{\prime}$ iff there exists some (possibly empty) sequence of events $\sigma \in \Sigma^{*}$ such that $h^{\prime}=h \sigma$, and write $\mathcal{K}_{i}[h]=\left\{h^{\prime} \mid h \sim_{i} h^{\prime}\right\}$. Let $H_{n}=\{h \mid \operatorname{len}(h)=n\}$ and $H_{\leq n}=\{h \mid \operatorname{len}(h) \leq n\}$. Given an ETL model $\mathcal{H}$ and $m<n, \mathcal{H} \backslash(m, n)$ is the restriction of the relations in $\mathcal{H}$ to $(H \times H) \backslash \bigcup_{1 \leq i \leq m}\left(\left(H_{i} \times H_{n}\right) \cup\left(H_{n} \times H_{i}\right)\right)$.

Definition 2. For an agent $i$ and two pointed models $\mathcal{M}, w$ and $\mathcal{M}^{\prime}, w^{\prime}$, we say that $w$ and $w^{\prime}$ are $i$ bisimilar, in symbols $w \simeq_{i} w^{\prime}$, iff

1. for each $v \in \mathcal{M}$ with $w \sim_{i} v$ there is $v^{\prime} \in \mathcal{M}^{\prime}$ such that $w^{\prime} \sim_{i} v^{\prime}$ and $v \simeq v^{\prime}$ 
2. for each $v^{\prime} \in \mathcal{M}$ with $w^{\prime} \sim_{i} v^{\prime}$ there is $v \in \mathcal{M}$ such that $w \sim_{i} v$ and $v \simeq v^{\prime}$,

where $\simeq$ denotes the standard epistemic bisimulation.

Because in this paper the role of preconditions will be trivialized by state-dependent protocols (Definition 3), we can proceed and state representation theorems independently of a choice of epistemic language. If we fix an epistemic language and a pointed model $\mathcal{M}, w$, we could formulate a notion of theory of agent $i$ at $w$ consisting of all statements of the form $\mathbf{K}_{i} \varphi$ and $\neg \mathbf{K}_{i} \varphi$ true at $w$ (where $\mathbf{K}_{i}$ is the knowledge operator for agent $i$ ). On finite frames, being $i$-bisimilar and having the same $i$-theory are equivalent.

Given two pointed ETL models $\mathcal{H}, h, \mathcal{H}^{\prime}, h^{\prime}$ and $m<$ $n$, we write that $\mathcal{H}, h \simeq_{i}{ }^{\backslash(m, n)} \mathcal{H}^{\prime}, h^{\prime}$ iff $\mathcal{H} \backslash(m, n), h \simeq_{i}$ $\mathcal{H}^{\prime} \backslash(m, n), h^{\prime}$. We sometimes write $h \simeq_{i}^{\mathcal{H} \backslash(m, n)} h^{\prime}$ to mean $\mathcal{H}, h \simeq_{i}{ }^{\backslash(m, n)} \mathcal{H}, h^{\prime}$.

As a last concept to be introduced, if $\left(e_{1} \ldots e_{n}\right) \in H$ is a history, we define agent $i$ 's experience record to be the sequence

$$
\mathrm{EE}_{i}\left(e_{1} \ldots e_{n}\right):=\mathcal{K}_{i}\left[e_{1}\right] \mathcal{K}_{i}\left[e_{1} e_{2}\right] \ldots \mathcal{K}_{i}\left[e_{1} \ldots e_{n}\right],
$$

and write $\mathrm{EE}_{i}\left(h_{1}\right) \approx_{i} \mathrm{EE}_{i}\left(h_{2}\right)$ iff $\mathrm{EE}_{i}\left(h_{1}\right)$ is equivalent to $\mathrm{EE}_{i}\left(h_{2}\right)$ up to stuttering.

In the following, we define a number of properties of ETL frames that will play an important role in our representation theorems.

We say that an S5 ETL model $\mathcal{H}=\left\langle\Sigma, H,\left(\sim_{i}\right)_{i \in N}, V\right\rangle$ satisfies

- Synchronicity (Syn) iff for all $i, h, h^{\prime}$ with $h \sim_{i}$ $h^{\prime}$ we have $\operatorname{len}(h)=\operatorname{len}\left(h^{\prime}\right)$

- Perfect recall (PR) iff for all $i, h, e, h^{\prime}$ with $h e \sim_{i}$ $h^{\prime}$ there is some $h^{\prime \prime} \leq h^{\prime}$ such that $h \sim_{i} h^{\prime \prime}$.

- Weak synchronous perfect recall (wsPR) iff for all $h, h^{\prime}, e, e^{\prime}$ with $h e \sim h^{\prime} e^{\prime}$, we have $h \sim h^{\prime}$.

- Synchronous perfect recall (sPR) iff for all $i, h, h^{\prime}, e, e^{\prime}$ with $h e \sim_{i} h^{\prime}$ there exists some $e^{\prime}$ and $h^{\prime \prime}$ such that $h^{\prime}=h^{\prime \prime} e^{\prime}$ and $h \sim_{i} h^{\prime \prime}$.

- Synchronous grounding (SG) iff for all $i, h, h^{\prime}$ with $h \sim_{i} h^{\prime}$ and $\operatorname{len}(h) \leq \operatorname{len}\left(h^{\prime}\right)$, there is some $h^{\prime \prime} \leq h^{\prime}$ with len $(h)=\operatorname{len}\left(h^{\prime \prime}\right)$ and $h \sim_{i} h^{\prime \prime}$.

- (Synchronous) uniform no miracles (UNM) iff for all $i$, if there are $h, h^{\prime}, e, f$ with $\operatorname{len}(h)=$ len $\left(h^{\prime}\right)$ and $h e \sim_{i} h^{\prime} f$, then for all $g, g^{\prime}$ such that $g \sim_{i} g^{\prime}$ and $\operatorname{len}(g)=\operatorname{len}\left(g^{\prime}\right)$ we have $g e \sim_{i} g^{\prime} f$.
- Weak uniform no miracles (wUNM) iff for all $i$, if there are $h, h^{\prime}, e, f$ with len $(h)=\operatorname{len}\left(h^{\prime}\right), h e \sim_{i}$ $h^{\prime} f$ and $h \chi_{i} h e$, then for all $g, g^{\prime}$ such that $g \sim_{i} g^{\prime}$ and len $(g)=\operatorname{len}\left(g^{\prime}\right)$ we have $g e \sim_{i} g^{\prime} f$.

- Perfect tracking (PT) iff for all $i, h, e, n$, if $h \sim_{i}$ $h e$ and len $(h)=n$ then $h \simeq_{i}^{\mathcal{H} \backslash(n, n+1)} h e$.

- No pure time perception (NPTP) iff for all $i, h, e, n$, if $h \simeq_{i}^{\mathcal{H} \backslash(n, n+1)} h e$ and $\operatorname{len}(h)=n$ then $h \sim_{i} h e$.

- Propositional stability (PS) iff for all $h, h^{\prime}$ and all $p \in$ PROP, if $h \leq h^{\prime}$ then $h \in V(p)$ iff $h^{\prime} \in V(p)$.

\subsection{The vBGHP representation}

We now give an account of the vBGHP representation. (Liu, 2008, Chapter 5) surveys earlier representation results. For a less compact presentation of the vBHGP representation the reader should consult van Benthem et al. 2009, §3).

Let $\mathfrak{E}$ be the class of all pointed event models; we let $\mathfrak{P}(\mathfrak{E}):=\left\{P \subseteq \mathfrak{E}^{*} \mid P\right.$ is closed under finite prefixes $\}$ be the set of all forests of sequences of event models.

Definition 3. Let $\mathcal{M}$ be an epistemic model. A statedependent protocol for $\mathcal{M}$ is a mapping $\mathrm{P}$ from $|\mathcal{M}|$ to $\mathfrak{P}(\mathfrak{E})$.

We define $\mathrm{P}_{\sigma}[\mathcal{M}]=\bigcup_{v \in|\mathcal{M}|} \mathrm{P}(v)$. Furthermore, $\mathrm{P}_{\mathrm{e}}[\mathcal{M}]=\left\{(\mathcal{E}, e) \mid(\mathcal{E}, e)\right.$ occurs in some $\left.\sigma^{*} \in \mathrm{P}_{\sigma}[\mathcal{M}]\right\}$.

The following two definitions are the foundations of the vBGHP representation:

Definition 4 (P-generated model, van Benthem et al. 2009) $)$. Let $\mathcal{M}=\left(W,\left(\sim_{i}\right)_{i \in N}, \bar{V}\right)$ be an epistemic model and $\mathrm{P}$ a state-dependent protocol for $\mathcal{M}$. The P-generated model at level $n, \mathcal{M}^{n, \mathrm{P}}=$ $\left\langle W^{n, \mathrm{P}},\left(\sim_{i}^{n, \mathrm{P}}\right)_{i \in N}, V^{n, \mathrm{P}}\right\rangle$ is inductively defined as follows:

- $\mathcal{M}^{0, \mathrm{P}}=\mathcal{M}$,

- $W^{n+1, \mathrm{P}}=\left\{w \sigma(\mathcal{E}, e) \mid w \sigma \in W^{n, \mathrm{P}}, \sigma(\mathcal{E}, e) \in\right.$ $\mathrm{P}(w)$ and $\left.\mathcal{M}^{n, \mathrm{P}}, w \sigma \Vdash \operatorname{pre}(\mathcal{E}, e)\right\}$,

- for every $w \sigma(\mathcal{E}, e), v \sigma^{\prime}\left(\mathcal{E}^{\prime}, e^{\prime}\right) \in W^{n+1, \mathrm{P}}$ we set $w \sigma(\mathcal{E}, e) \sim_{i}^{n+1, \mathrm{P}} v \sigma^{\prime}\left(\mathcal{E}^{\prime}, e^{\prime}\right)$ iff $\mathcal{E}=\mathcal{E}^{\prime}, e \sim_{i}^{\mathcal{E}} e^{\prime}$ $w \sigma \sim_{i}^{n, \mathrm{P}} v \sigma^{\prime}$,

- and, for every $w \sigma(\mathcal{E}, e) \in W^{n+1, \mathrm{P}}$ and $p \in \mathrm{PROP}$, we set $w \sigma(\mathcal{E}, e) \in V^{n+1, \mathrm{P}}(p)$ iff $w \sigma \in V^{n, \mathrm{P}}(p)$.

Definition 5 (Synchronously DEL generated ETL models, $\operatorname{van} B$ Benthem et al. 2009). Let $\mathcal{M}=$ $\left(W,\left(\sim_{i}\right)_{i \in N}, V\right)$ be an epistemic model and $\mathrm{P}$ be a 
state-dependent DEL protocol for $\mathcal{M}$, the ETL model synchronously generated by $\mathcal{M}$ and $\mathrm{P}$ is defined as

$$
\operatorname{sForest}(\mathcal{M}, \mathrm{P}):=\left(\Sigma, H,\left\{\sim_{i}\right\}_{i \in N}, V^{\prime}\right),
$$

where

1. $\Sigma=\mathrm{P}_{\mathrm{e}}[\mathcal{M}] \cup|\mathcal{M}|$,

2. $H=\bigcup_{n \geq 0} W^{n, P}$,

3. for all $h, h^{\prime} \in H \cap W, h \sim h^{\prime}$ iff $h \sim_{i}^{0, \mathrm{P}} h^{\prime}$.

4. for all $h, h^{\prime} \in H \backslash W$ with $h=w \sigma$ and $h^{\prime}=v \sigma^{\prime}$, $h \sim_{i} h^{\prime}$ iff $\operatorname{len}(\sigma)=\operatorname{len}\left(\sigma^{\prime}\right)$ and $h \sim_{i}^{\operatorname{len}(\sigma), \mathrm{P}} h^{\prime}$,

5. and, for all $p \in$ PROP, $h \in V^{\prime}(p)$ iff $h \in$ $V^{\operatorname{len}(h-1), \mathrm{P}}(p)$.

Note that we denote the translation function from (van Benthem et al. 2009) by sForest (for "synchronously DEL generated ETL Forest") in order to distinguish it from our later notion of asForest (for "asynchronously DEL generated ETL Forest"). Using Definitions 4 and 5. we can now state the vBGHP representation:

Theorem 6 (van Benthem, Gerbrandy, Hoshi, Pacuit). For an S5 epistemic-temporal ETL model, the following are equivalent:

1. $\mathcal{H}$ is isomorphic to $\operatorname{sForest}(\mathcal{M}, \mathrm{P})$ for some epistemic model $\mathcal{M}$ and some state-dependent DEL protocol $\mathrm{P}$, and

2. $\mathcal{H}$ satisfies wsPR, Syn, UNM, and PS.

\section{The issue of perfect recall}

The reader might wonder why we have three different notions of perfect recall in our list of properties of ETL models: PR, sPR and wsPR. The common formulation of perfect recall from the interpreted systems literature is our PR (Fagin et al. 1995) ${ }^{2}$ It is however, not the definition of perfect recall in the vBGHP representation. As can be seen in Theorem 6, the authors of van Benthem et al. (2009) use wsPR instead ${ }^{3}$

Observation 7. On synchronous S5 ETL models, PR, wsPR, and sPR are equivalent.

Proof. Cf. (Witzel, 2011).

\footnotetext{
${ }^{2}$ More precisely, it is a slight and equivalent variant of the formulation in (Halpern et al., 2004 Lemma 2.2(d)).

${ }^{3}$ sPR was the notion of perfect recall used in the earlier representation results (van Benthem, 2001, 2006).
}

However, in general, PR does not imply wsPR (see below, Proposition 11). While PR has intuitive interpretations (Witzel, 2011, where PR is called PR $\mathrm{hc}_{\mathrm{hc}}$ ), wsPR is used as a technical notion without intuitive motivation; only if one presupposes synchronicity, wsPR inherits the intuition from PR. For this reason, we use $\mathrm{PR}$ as the fundamental definition of perfect recall.

Observation 8. On synchronous S5 ETL models, UNM and wUNM are equivalent.

Using Observations 7 and 8 and the fact that SG and PT are trivially true on synchronous models, we can now restate the vBGHP representation in terms of PR.

Corollary 9. For an S5 epistemic-temporal ETL model, the following are equivalent:

1. $\mathcal{H}$ is isomorphic to sForest $(\mathcal{M}, \mathrm{P})$ for some epistemic model $\mathcal{M}$ and some state-dependent DEL protocol $\mathrm{P}$, and

2. $\mathcal{H}$ satisfies PR, Syn, PT, SG, wUNM, and PS.

We end this section by introducing two equivalent alternative characterizations of PR, which will be convenient for some of our later proofs. The first one is a natural notion of perfect recall very close in spirit to the game-theoretic one (Osborne and Rubinstein, 1994, Chapter 11).

Definition 10. We say that an ETL model $\mathcal{H}=$ $\left\langle\Sigma, H,\left(\sim_{i}\right)_{i \in N}, V\right\rangle$ satisfies

- perfect recall based on epistemic experience $\left(\mathrm{PR}_{\mathrm{ee}}\right)$ iff for all $i, h, h^{\prime}$ with $h \sim_{i} h^{\prime}$, we have $\operatorname{EE}_{i}(h) \approx_{i} \operatorname{EE}_{i}\left(h^{\prime}\right)$.

- perfect recall, local version $\left(\mathrm{PR}^{\ell}\right)$ iff for each $i, h, h^{\prime}, e$ with $h e \sim_{i} h^{\prime}$, one of the following holds:

(i) $h \sim_{i} h^{\prime}$

(ii) $h \sim_{i} h^{\prime \prime}$

(iii) $h e \sim_{i} h^{\prime \prime}$

where $h^{\prime \prime}$ is the direct predecessor of $h^{\prime}$, i.e., $h^{\prime}=$ $h^{\prime \prime} e^{\prime}$ for some $e^{\prime}$.

The following result states the relationships between the various versions of perfect recall:

Proposition 11. On S5 ETL models, PR, PR ee and $\mathrm{PR}^{\ell}$ are equivalent; wsPR neither implies nor is implied by these; and sPR is equivalent to synchronicity plus PR. On synchronous S5 ETL models, all of the notions are equivalent.

Proof. Cf. (Witzel, 2011). Figure 1 is also taken from (Witzel, 2011) and provides the examples that show that PR and wsPR do not imply each other (the relevant part of the claim of Proposition 11 for our present purposes). 


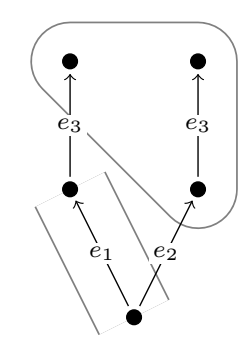

(a) PR, but not wsPR

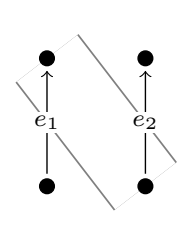

(b) wsPR, but not

PR

Figure 1: Two S5 ETL models, gray lines indicating information sets (reflexive loops omitted). (a) PR does not imply wsPR (wsPR is violated because $e_{1} e_{3} \sim e_{2} e_{3}$ but $e_{1} \nsim e_{2}$ ), and (b) wsPR does not imply PR on ETL forests: Intuitively, event $e_{1}$ lets the agent "forget" that he is in the left tree.

\section{Asynchronous ETL models and our representation theorem}

In this section, we give our alternative translation from DEL to ETL that will in general produce asynchronous ETL models.

Definition 12 (Asynchronously DEL generated ETL models). Let $\mathcal{M}=\left(W,\left(\sim_{i}\right)_{i \in N}, V\right)$ be an epistemic model and $\mathrm{P}$ be a state-dependent DEL protocol for $\mathcal{M}$, the ETL model asynchronously generated by $\mathcal{M}$ and $P$ is defined as

$$
\operatorname{asForest}(\mathcal{M}, \mathrm{P}):=\left(\Sigma, H,\left\{\sim_{i}^{\prime}\right\}_{i \in N}, V\right),
$$

where $\left(\Sigma, H,\left\{\sim_{i}\right\}_{i \in N}, V\right)=\operatorname{sForest}(\mathcal{M}, \mathrm{P})$, and where $\sim_{i}^{\prime}$ is the symmetric transitive closure of

$$
\begin{array}{r}
\sim_{i} \cup\{(w \sigma, w \sigma(\mathcal{E}, e)) \in(H \times H) \mid \sigma(\mathcal{E}, e) \in \mathrm{P}(w) \\
\text { and } \left.w \sigma \simeq_{i}^{\text {sForest }(\mathcal{M}, \mathrm{P})} w \sigma(\mathcal{E}, e)\right\} .
\end{array}
$$

(asForest stands for "asynchronously DEL generated ETL Forest".)

To get a feeling for the definition of asForest, let us consider the simplest possible example: an epistemic model $\mathcal{M}$ consisting of one world $w$, and the trivial event model $\mathcal{E}$ consisting of one event $e$ with precondition $T$. Then $\mathcal{M}$ and $\mathcal{M} \otimes \mathcal{E}$ are isomorphic, thus bisimilar, and therefore $w \simeq_{i} w e$. In the ETL model $\operatorname{asForest}(\mathcal{M}, \mathrm{P})$, we have two elements, $w$ and $w e$, and we know that $w \sim_{i}^{\prime} w e$, violating synchronicity.

We now give a more elaborate example:

Example 13. The model $\mathcal{M}$ in Figure 2 represents a situation with two agents $i, j$ in which neither agent knows the truth value of a proposition $p$, but $i$ considers it possible that $j$ does know either $p$ or $\neg p$ (and all this is common knowledge). The event model $\mathcal{E}$ with domain $\left\{\left(e_{1}, e_{2}\right)\right\}$, with $\sim_{j}$ being the minimal reflexive relation and $\sim_{i}$ the universal relation, and with $\operatorname{pre}\left(e_{1}\right)=p$ and $\operatorname{pre}\left(e_{2}\right)=\top$ represents the event in which agent $j$ learns that $p$ is true $\left(e_{1}\right)$, but agent $i$ considers it possible that nothing happens $\left(e_{2}\right)$. In the resulting model $\mathcal{M} \otimes \mathcal{E}, j$ knows $p$, while agent $i$ 's knowledge has not changed, since he already before considered it possible that $j$ actually knew $p$. All of this is represented in Figure 2
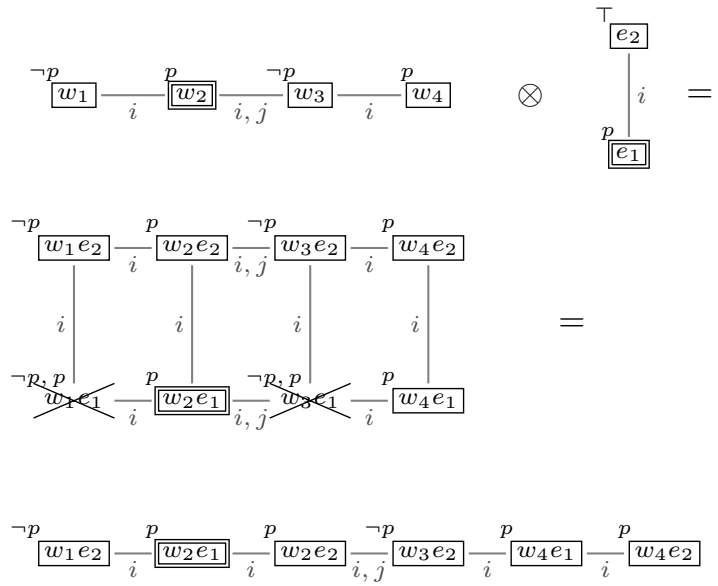

Figure 2: DEL model $\mathcal{M}$, event model $\mathcal{E}$, and resulting model $\mathcal{M} \otimes \mathcal{E}$, first shown to illustrate the product operation and then transformed into a line. Transitive and reflexive accessibilities omitted.

For any $w \in|\mathcal{M}|$, let $\mathrm{P}(w)=\left\{\left(\mathcal{E}, e_{1}\right),\left(\mathcal{E}, e_{2}\right)\right\}$. We now apply sForest to obtain an ETL model as given in Figure 3. By design, sForest $(\mathcal{M}, \mathrm{P})$ just consists of $\mathcal{M} \otimes \mathcal{E}$ stacked on top of $\mathcal{M}$.

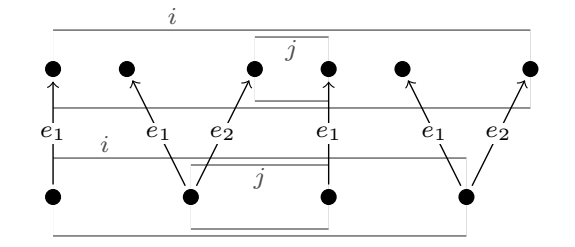

Figure 3: $\operatorname{sForest}(\mathcal{M}, \mathrm{P})$

If we inspect the epistemic model, we realize that $w_{2} \simeq_{i} w_{2} e_{2}, w_{3} \simeq_{i} w_{3} e_{1}, w_{2} \simeq_{j} w_{2} e_{2}$, and $w_{3} \simeq_{j}$ $w_{3} e_{1}$. Using this fact for the definition of the equivalence relations $\sim_{i}^{\prime}$ and $\sim_{j}^{\prime}$, we obtain the ETL model given in Figure 4

The event $\mathcal{E}$ represents that $j$ gains some information, but $i$ does not know whether $j$ gains this information; it does not specify whether $i$ does not realize that anything is happening at all. Whether this is the case, depends on the situation that is being modelled: if $i$ sees $j$ talk to some other agent who knows 


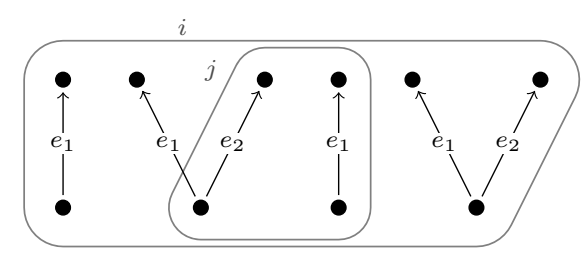

Figure 4: $\operatorname{asForest}(\mathcal{M}, \mathrm{P})$

whether $p$, but cannot hear the conversation, one may argue that $i$ does realize that something is happening even though his epistemic state as represented in the model is not changing; in this case sForest is more appropriate. On the other hand, if $i$ cannot see this conversation, then asForest represents this state of affairs better than sForest.

The event model itself does not distinguish between these two intended interpretations. In Section 6, we therefore propose a slight extension of DEL models that will allow for this distinction 4

As a first property of our proposed translation, we prove that asForest satisfies perfect recall.

Proposition 14. For any model $\mathcal{M}$ and DEL protocol $\mathrm{P}$, asForest $(\mathcal{M}, \mathrm{P})$ has perfect recall.

Proof. First we note that $\operatorname{sForest}(\mathcal{M}, \mathrm{P})$ has perfect recall. For any two histories $h_{1}, h_{2}$ and events $e_{1}, e_{2}$, the product update allows $h_{1} e_{1} \sim h_{2} e_{2}$ only if $h_{1} \sim h_{2}$, so PR is satisfied.

The rest of the proof uses the (equivalent) definition $\mathrm{PR}_{\mathrm{ee}}$ for perfect recall. That is, we show that in any asForest, whenever $h_{1} \sim_{i} h_{2}$, then $\operatorname{EE}\left(h_{1}\right) \approx_{i} \operatorname{EE}\left(h_{2}\right)$.

We start by showing that equivalence of epistemic experiences modulo stutterings is preserved under joining information sets. Formally, for any agent $i$, consider any two histories $h_{1}, h_{2}$ of some forest with accessibility relation $\sim_{i}$ which has $\mathrm{EE}_{i}\left(h_{1}\right) \approx_{i} \mathrm{EE}_{i}\left(h_{2}\right)$. Then in the forest that is obtained by letting $h_{1}^{\prime} \sim_{i} h_{2}^{\prime}$ for any two histories $h_{1}^{\prime}, h_{2}^{\prime}$ and closing $\sim_{i}$ off under symmetry and transitivity, we still have $\mathrm{EE}_{i}\left(h_{1}\right) \approx_{i}$ $\mathrm{EE}_{i}\left(h_{2}\right)$.

To see this, first recall that $i$ 's epistemic experience $\mathrm{EE}_{i}(h)$ at some history $h$ is the sequence of information

\footnotetext{
${ }^{4}$ The distinction between internal and external perspective (Aucher, 2008) becomes relevant here: if we view DEL as "internal reasoning engine" of some (artificial) agent, the fact that that agent even updates the current model means that he was notified in some way about the event, even if the update has no effect on his represented epistemic state; in that case, only sForest seems adequate. If we assume the viewpoint of an external modeler who keeps track of agents' epistemic states as events occur, asForest becomes plausible.
}

sets he has gone through. Joining the two information sets $\left[h_{1}^{\prime}\right]_{\sim_{i}}$ and $\left[h_{2}^{\prime}\right]_{\sim_{i}}$ results in replacing occurrences of either of these two sets in that sequence by one and the same set $\left[h_{1}^{\prime}\right]_{\sim_{i}} \cup\left[h_{2}^{\prime}\right]_{\sim_{i}}$. The relation $\approx_{i}$, equivalence modulo stutterings, can thus only grow when joining information sets.

We now turn to the main claim. We show that adding a single "vertical" accessibility $h \sim_{i}$ he for some history $h$ and event $e$ and closing off under symmetry and transitivity preserves perfect recall. The main claim then follows inductively since asForest $(\mathcal{M}, \mathrm{P})$ is obtained from $s$ Forest $(\mathcal{M}, \mathrm{P})$ by several such operations and, as noted above, $\operatorname{sForest}(\mathcal{M}, \mathrm{P})$ has perfect recall.

So let $\mathcal{H}$ denote some S5 ETL model with perfect recall, $h$ and $h e$ some histories in $\mathcal{H}$, and $\mathcal{H}^{\prime}$ the ETL model with $h \sim_{i}$ he added and closed off. For any $h_{1}, h_{2}$ with $h_{1} \sim_{i} h_{2}$ already in $\mathcal{H}$, we have $\mathrm{EE}_{i}\left(h_{1}\right) \approx_{i} \mathrm{EE}_{i}\left(h_{2}\right)$ in $\mathcal{H}$ since $\mathcal{H}$ has perfect recall. As established above, the relation $\approx_{i}$ can only grow when joining information sets, and so we have $\operatorname{EE}_{i}\left(h_{1}\right) \approx_{i} \operatorname{EE}_{i}\left(h_{2}\right)$ also in $\mathcal{H}^{\prime}$.

It remains to show that the condition of $\mathrm{PR}_{\mathrm{ee}}$ is satisfied for the accessibilities added in $\mathcal{F}^{\prime}$. For the explicitly added accessibility $h \sim_{i} h e$, we have $[h]_{\sim_{i}}=[h e]_{\sim_{i}}$ in $\mathcal{F}^{\prime}$ and thus $\operatorname{EE}_{i}(h) \approx_{i} \mathrm{EE}_{i}(h e)$ since $\approx_{i}$ disregards stutterings. For the accessibilities added by closing off under symmetry and transitivity, the condition is satisfied by symmetry and transitivity of $\approx_{i}$.

Lemma 15. For any ETL model $\mathcal{H}$ with perfect recall and synchronous grounding, and for any two histories $h e$ and $h^{\prime} e^{\prime}$, if len $(h)=\operatorname{len}\left(h^{\prime}\right)$ and $h e \sim h^{\prime} e^{\prime}$ then $h \sim h^{\prime}$.

Proof. Since $\mathrm{PR}^{\ell}$ characterizes perfect recall, we obtain one of these three cases:

(i) $h \sim h^{\prime} e^{\prime}$, then $h \sim h^{\prime}$ follows from synchronous grounding;

(ii) $h \sim h^{\prime}$, done;

(iii) $h e \sim h^{\prime}$, then $h \sim h^{\prime}$ again follows from synchronous grounding.

We are finally ready to state our main theorem.

Theorem 16. For an S5 epistemic-temporal ETL model, the following are equivalent:

1. $\mathcal{H}$ is isomorphic to asForest $(\mathcal{M}, \mathrm{P})$ for some epistemic model $\mathcal{M}$ and some state-dependent DEL protocol $\mathrm{P}$, and

2. $\mathcal{H}$ satisfies PR, NPTP, PT, SG, wUNM, and PS. 
Compare this to Corollary 9 where sForest is characterized in terms of the same properties, except that NPTP is replaced by Syn. The proof can be found in Appendix A.

\section{Relating the two representation theorems}

We should stress that there is no conflict between the two representation theorems (the two embeddings are different, hence so are the epistemic temporal properties), and that none of them is a generalization of the other. The two translations sForest or asForest are opposite extremes of interpreting the ambiguity in the process of translating temporally underspecified DEL models into ETL models.

Exploring these two extremes can help us to understand better which of the temporal properties we investigated are core DEL properties and which ones are properties that depend on the choice of the embedding. Properties shared by the two embeddings are candidates for core DEL properties. We briefly discuss the various properties involved, discussing their degree of entrenchment in DEL, their desirability (for flexible formalisms for modelling multi-agent systems), and possibilities of lifting them. Of course, questions of desirability as well as questions of whether a given embedding is natural are non-mathematical questions and will not be answered definitively.

Synchronicity. As demonstrated through the two translations, DEL can be interpreted as either satisfying Syn or NPTP. These are diametrically opposed properties of a range of conceivable perceptions of time. Section 6 shows how DEL can be extended to explicitly distinguish between these two extremes and the whole range between them.

Perfect recall is a consequence of the way the DEL product update works: intuitively, it can only remove epistemic accessibilities. With respect to what was the case before the event happened, product update can only reduce uncertainty, thus can only increase knowledge. This monotonicity of informational states, under new update by new events, is precisely what doxastic versions of DEL using priority update (Baltag and Smets, 2006. van Benthem, 2007) weaken, enabling agents to truly revise their beliefs (cf. $\S 7$ ).

Synchronous grounding reflects the fact that product update is applied uniformly to the whole model. This seems rather deeply entrenched in DEL, and defining updates that are only applied to parts of a model seems difficult. An interpretation of the property is that if Alice considers possible some history $h$ that she is no longer synchronous with, it must because when she was still synchronous with it, she was already considering $h$ possible.

Weak uniform no miracles (like its stronger version) reflects the fact that product update is a local, i.e. history-independent, update.

Perfect tracking. The fact that successive states of the world can only be indistinguishable if an agent's epistemic state has not changed is natural and justified: it is analogous to standard epistemic introspection assumptions.

Propositional stability is (as is well-known) a serious restriction and can be removed by considering ontic actions (see, e.g., van Benthem et al. 2006)).

\section{A minimal temporal extension of DEL}

A natural question is thus how to enable DEL to distinguish between classes of ETL models assuming the mentioned extremal perceptions of time, and any intermediate ones. We can do so using a very simple idea: a history-dependent clock ticking function. This minimalistic extension of protocols-based DEL removes the temporal ambiguity and enables DEL to distinguish, at any point in time, between the asynchronous and the synchronous interpretation 5

Our proposed extension enriches DEL protocols with flags, one for each agent, specifying whether or not a new epistemic situation is (potentially) indistinguishable from the old situation for that specific agent. Intuitively, this can be thought of as specifying whether or not an agent hears a clock tick as this event occurs. In terms of epistemic experience, this flag specifies whether a stuttering resulting from this atomic event should be conflated or not.

We make this idea precise in the following sequence of definitions.

Definition 17. Given a state dependent protocol $P$ for $\mathcal{M}$ a history dependent clock tick function is a function $\mathrm{c}:\left(|\mathcal{M}| \times \mathrm{P}_{\sigma}[\mathcal{M}] \times \mathfrak{E}\right) \rightarrow 2^{N}$.

E.g., we interpret $\mathrm{c}(w, \sigma,(\mathcal{E}, e))=\{i\}$ to mean

\footnotetext{
${ }^{5}$ Of course, more sophisticated extensions of DEL that account for synchronous and asynchronous scenarios are possible, as the one proposed by Renne et al. (2009). We are aiming here for the minimal extension that does the job.
} 
if the initial state was $w$, the sequence $\sigma$ of events has occurred, and $(\mathcal{E}, e)$ happens, then agent $i$, even if his or her epistemic state remains the same, will notice that time has passed; and no one else does.

Now we turn to the construction.

Definition 18 (clock tick function DEL generated ETL models). Let $\mathcal{M}=\left(W,\left(\sim_{i}\right)_{i \in N}, V\right)$ be an epistemic model and $\mathrm{P}$ be a state-dependent DEL protocol with a clock tick function $\mathrm{c}$, the ETL model generated by $\mathcal{M}, \mathrm{P}$ and $\mathrm{c}$ is defined as

$$
\operatorname{clickForest}(\mathcal{M}, \mathrm{P}, \mathrm{c}):=\left(\Sigma, H,\left\{\sim_{i}^{\prime}\right\}_{i \in N}, V\right) \text {, }
$$

where $\left(\Sigma, H,\left\{\sim_{i}\right\}_{i \in N}, V\right)=\operatorname{sForest}(\mathcal{M}, \mathrm{P})$, and where $\sim_{i}^{\prime}$ is the symmetric transitive closure of

$$
\begin{aligned}
\sim_{i} \cup\{ & (w \sigma, w \sigma(\mathcal{E}, e)) \in(H \times H) \mid \\
& \sigma(\mathcal{E}, e) \in \mathrm{P}(w) \\
& \text { and } w \sigma \simeq_{i}^{\operatorname{sForest}(\mathcal{M}, \mathrm{P})} w \sigma(\mathcal{E}, e) \\
& \text { and } i \notin \mathrm{c}(w, \sigma,(\mathcal{E}, e))\} .
\end{aligned}
$$

(clickForest stands for "clock tick function DEL genrated ETL Forest".)

We now give a representation theorem that accounts for the whole class of ETL models having the mentioned DEL-originated epistemic-temporal properties.

Theorem 19. For an S5 epistemic-temporal ETL model, the following are equivalent:

1. $\mathcal{H}$ is isomorphic to $\operatorname{clickForest}(\mathcal{M}, \mathrm{P}, \mathrm{c})$ for some epistemic model $\mathcal{M}$, some state-dependent DEL protocol $\mathrm{P}$ and some clock tick function $\mathrm{c}$.

2. $\mathcal{H}$ satisfies perfect recall, perfect tracking, synchronous grounding, synchronous uniform no miracles and propositional stability.

The proof can be found in Appendix B.

Examples. The empty event (one atomic event $e$ with precondition $T$ ) can now be used to represent events of pure (loss of) synchronicity among agents, depending on the clock tick function c. If c $(e)=A \subseteq N$, the event has the effect that all agents in $A$ stay "in sync", while all others get "out of sync".

Another practical use of this extension is for incorporating a form of agency into DEL events, bringing it closer in spirit to game theoretic or autonomous agent frameworks. In those frameworks, events are predominantly taken to be a particular agent's actions, and the actions performed by an agent are assumed to be part of the local state description (in case of perfect recall agents), so that agents can always distinguish between states of the world in which the sequence of their own actions differs. We can now specify which (atomic or composite) events constitute an agent's set of actions by setting the flag so that he "hears a clock tick" whenever he performs one of his actions, thus ensuring that he can discern whether and how he has acted even if his epistemic state (in terms of the DEL model) doesn't change.

\section{Conclusions and future work}

The constructions given in Definition 12 (without clock tick function) and Definition 18 (with clock tick function) can generate asynchronous models from statedependent DEL protocols. But it should be noted that the diachronic uncertainties generated this way are of a very special type. In particular, (non-trivial) asynchronicities are only obtained if an agent already "happens to" have anticipated the possibility of an event occurring. Put differently, there is no (non-trivial) event that inherently gives rise to asynchronicity; it always depends on a specific situation.

This observation is not unexpected; after all, we are dealing with S5 knowledge: Agents cannot be mistaken, so if an event occurs that they do not notice, they must already have considered its consequences possible. However, for modeling realistic multi-agent systems in which agents may be really separate entities and make private observations, this is a severe restriction.

Future work. To account for a wider ranger of diachronic uncertainties and more general situations, two natural directions can be explored. The first one is to give up S5, i.e., weaken the assumption that epistemic accessibility relations are equivalence relations. The second is to consider richer models that include both an epistemic accessibility relation, encoding agents' information, and a plausibility ordering, indicating which states (or histories) are a priori more likely, taking beliefs to be defined as 'true in the most plausible states in the current information set'.

In the first direction it might be the case that some of the previous properties would need to be adjusted, were we to drop the assumptions that we are working with equivalence relations, i.e., with partitions. It is however difficult to make any conjecture, since the $\mathrm{S} 5$ assumption plays a role in numerous places in our proofs. Moreover the natural symmetry with the synchronous case would probably be lost. As an example, recall from Proposition 11 that if we drop the S5 assumption different notions of perfect recall start to become incomparable. This contrasts drastically with the situation in the synchronous case: indeed the 
vBGHP representation do not require any of the transitivity, reflexivity or symmetry assumption.

In the second direction, epistemic models are enriched into epistemic-plausibility models (Board, 2004, van Ditmarsch, 2005, Baltag and Smets, 2006 van Benthem, 2007). Similarly ETL models can be enriched and this has lead to similar synchronous representation results (van Benthem and Dégremont, 2010. Dégremont, 2010). Now, it is not immediate to decide what would be the most natural way to extend the idea behind our asynchronous construction to this richer setting. Indeed, the intuition of the asychronous construction was that "agents can only know that some event has happened, if their epistemic state has changed". We could adapt it as follow "agents can only believe that some events happened, if their doxastic state has changed", leading to the idea that by default, if nothing has change in the doxastic state of the agent after some event, then she should still consider it most plausible that nothing has happened. This could account for scenarios in which agents have opposite beliefs about whether some event has happened or not.

\section{Acknowledgements}

The research of the first and second author was partially supported by the Early Stage Research Training Mono-Host Fellowship GLoRiClass funded by the European Commission (MEST-CT-2005-020841). This project started while the second author was visiting the CUNY Graduate Center in New York in the fall of 2009; he expresses his gratitude for the hospitality of his hosts.

\section{References}

Guillaume Aucher. Perspectives on Belief and Change. PhD thesis, Université de Toulouse \& University of Otago, 2008.

Alexandru Baltag and Sonja Smets. Dynamic belief revision over multi-agent plausibility models. In G. Bonanno, W. van der Hoek, and M. Wooldridge, editors, Logic and the Foundations of Game and Decision Theory (LOFT 7), volume 3 of Texts in Logic and Games, pages 11-24. Amsterdam University Press, 2006.

Alexandru Baltag, Lawrence S. Moss, and Slawomir Solecki. The logic of public announcements, common knowledge, and private suspicions. In Itzhak Gilboa, editor, Proceedings of the 7th Conference on Theoretical Aspects of Rationality and Knowledge (TARK-98), pages 43-56, San Francisco, CA, USA, 1998. Morgan Kaufmann Publishers Inc.
Oliver Board. Dynamic interactive epistemology. Games and Economic Behavior, 49:49-80, 2004.

Cédric Dégremont. The Temporal Mind. Observations on the logic of belief change in interactive systems. $\mathrm{PhD}$ thesis, Universiteit van Amsterdam, 2010. ILLC Publications DS-2010-03.

Ronald Fagin, Joseph Y. Halpern, Moshe Y. Vardi, and Yoram Moses. Reasoning About Knowledge. MIT Press, Cambridge, 1995.

Joseph Y. Halpern, Ron van der Meyden, and Moshe Y. Vardi. Complete axiomatizations for reasoning about knowledge and time. SIAM Journal on Computing, 33(3):674-703, 2004.

Tomohiro Hoshi. Epistemic Dynamics and Protocol Information. PhD thesis, Stanford University, 2009. ILLC Publications DS-2009-08.

Alistair Isaac and Tomohiro Hoshi. Synchronizing diachronic uncertainty. Journal of Logic, Language and Information, to appear.

Fenrong Liu. Changing for the Better: Preference Dynamics and Agent Diversity. $\mathrm{PhD}$ thesis, Universiteit van Amsterdam, 2008. ILLC Publications DS2008-02.

Martin J. Osborne and Ariel Rubinstein. A Course in Game Theory. MIT Press, 1994.

Rohit Parikh and Ramaswamy Ramanujam. A knowledge based semantics of messages. Journal of Logic, Language and Information, 12(4):453-467, 2003.

Bryan Renne, Joshua Sack, and Audrey Yap. Dynamic epistemic temporal logic. In Xiangdong He, John Horty, and Eric Pacuit, editors, Logic, Rationality, and Interaction. Second International Workshop, LORI 2009, Chongqing, China, October 8-11, 2009, Proceedings, volume 5834 of Lecture Notes in Artificial Intelligence, pages 263-277. Springer, 2009.

Joshua Sack. Adding temporal logic to dynamic epistemic logic. PhD thesis, Indiana University, 2007.

Johan van Benthem. Games in Dynamic Epistemic Logic. Bulletin of Economic Research, 53(4):219248, 2001.

Johan van Benthem. One is a lonely number. In Z. Chatzidakis, P. Koepke, and W. Pohlers, editors, Logic Colloquium '02. Joint proceedings of the Annual European Summer Meeting of the Association for Symbolic Logic and the Biannual Meeting of the German Association for Mathematical Logic and the Foundations of Exact Sciences (the Colloquium Logicum) held in Münster, volume 27 of Lecture Notes in Logic, Wellesley MA, 2006. ASL \& A.K. Peters.

Johan van Benthem. Dynamic logic for belief revision. Journal of Applied Non-classical Logics, 17(2), 2007. 
Johan van Benthem and Cédric Dégremont. Bridges between dynamic doxastic and doxastic temporal logics. In Giacomo Bonanno, Benedikt Löwe, and Wiebe van der Hoek, editors, Logic and the Foundations of Game and Decision Theory (LOFT 8), volume 6006 of Lecture Notes in Artificial Intelligence, pages 151-173. Springer, 2010.

Johan van Benthem, Jan van Eijck, and Barteld Kooi. Logics of communication and change. Information and Computation, 204(11):1620-1662, 2006.

Johan van Benthem, Jelle Gerbrandy, Tomohiro Hoshi, and Eric Pacuit. Merging frameworks for interaction. Journal of Philosophical Logic, 38(5): 491-526, 2009.

Wiebe van der Hoek and Michael Wooldridge. Cooperation, knowledge, and time: Alternating-time temporal epistemic logic and its applications. Studia Logica, 75(1):125-157, 2003.

Hans van Ditmarsch. Prolegomena to dynamic logic for belief revision. Synthese, 147:229-275, 2005.

Hans van Ditmarsch, Wiebe van der Hoek, and Barteld Kooi. Dynamic Epistemic Logic. Springer, 2007.

Yanjing Wang, Floor Sietsma, and Jan van Eijck. Logic of information flow on communication channels (extended abstract). In Wiebe van der Hoek, Gal A. Kaminka, Yves Lespérance, Michael Luck, and Sandip Sen, editors, Proceedings of the 9th International Conference on Autonomous Agents and Multi-Agent Systems (AAMAS 2010), pages 14471448, 2010.

Andreas Witzel. Characterizing perfect recall in epistemic temporal logic. Journal of Logic and Computation, 2011. Conditionally accepted.

\section{APPENDIX}

\section{APPENDIX}

\section{A Proof of Theorem 16.}

A piece of notation: given an ETL model $\mathcal{H}, \mathcal{H} \mid n$ is the restriction of $\mathcal{H}$ to $H_{n}, \mathcal{H} \mid \leq n$ is the restriction of $\mathcal{H}$ to $H_{\leq n}$.

Observation 20. Let $\mathcal{H}=\left\langle\Sigma, H,\left(\sim_{i}\right)_{i \in N}, V\right\rangle$ be an S5 ETL model and let $\left(h e, h^{\prime} f\right) \in H_{n+1} \times H_{n+1}$ be such that $h \sim_{i} h^{\prime}$, and $\mathcal{H}^{k}, h \simeq_{i} \backslash(n, n+1) \quad \mathcal{H}^{k}$, he and $\mathcal{H}^{k}, h^{\prime} \simeq_{i}^{\backslash(n, n+1)} \mathcal{H}^{k}, h^{\prime} f$. We have $\mathcal{H}^{k}, h e \simeq_{i} \backslash(n, n+1)$ $\mathcal{H}^{k}, h^{\prime} f$.

Definition 21. Let $\mathcal{H}=\left\langle\Sigma, H,\left(\sim_{i}\right)_{i \in N}, V\right\rangle$ and $\mathcal{H}^{\prime}=$ $\left\langle\Sigma^{\prime}, H^{\prime},\left(\sim_{i}^{\prime}\right)_{i \in N}, V^{\prime}\right\rangle$ be two S5 ETL models. We say that $\mathcal{H}^{\prime}$ is $n+1$-bisimulation closure reachable from $\mathcal{H}$ iff the following conditions hold:

1. $\Sigma=\Sigma^{\prime} ; H=H^{\prime}$; for every $h \in H$ and $p \in$ PROP $h \in V(p)$ iff $h \in V^{\prime}(p)$

2. for every $i \in N$ and $\left(h, h^{\prime}\right) \in(H \times H) \backslash\left(H_{n+1} \times\right.$ $H_{n+1}$ ) we have $h \sim_{i} h^{\prime}$ iff $h \sim_{i}^{\prime} h^{\prime}$

3. for every $i \in N, \sim_{i} \subseteq \sim_{i}^{\prime}$

4. there exists a sequence of ETL models $\mathcal{H}^{0} \ldots \mathcal{H}^{m}$ with $\mathcal{H}^{0}=\mathcal{H}, \mathcal{H}^{m}=\mathcal{H}^{\prime}$ such that for all $k$ with $0 \leq k<m$, we have $\mathcal{H}^{k}=\left\langle\Sigma, H,\left(\sim_{i}^{k}\right)_{i \in N}, V\right\rangle$ and there exists $i \in N$ and there exists a pair of histories $\left(h e, h^{\prime} f\right) \in H_{n+1} \times H_{n+1}$ with $\mathcal{H}^{k}, h e \simeq_{i}{ }^{\backslash(n, n+1)} \mathcal{H}^{k}, h^{\prime} f$ such that $\sim_{i}^{k+1}$ is the reflexive, transitive and symmetric closure of $\sim_{i}^{k}$ $\cup\left\{\left(h e, h^{\prime} f\right)\right\}$ and for every $j \in N \backslash\{i\}, \sim_{j}^{k+1}=\sim_{j}^{k}$.

The following is a general fact about S5 ETL models, that will be useful in this paper.

Fact 22. Let $\mathcal{H}=\left\langle\Sigma, H,\left(\sim_{i}\right)_{i \in N}, V\right\rangle$ and $\mathcal{H}^{\prime}=$ $\left\langle\Sigma^{\prime}, H^{\prime},\left(\sim_{i}^{\prime}\right)_{i \in N}, V^{\prime}\right\rangle$ be two S5 ETL models with $\mathcal{H}^{\prime}$ $n+1$-bisimulation closure reachable from $\mathcal{H}$. We claim that for all $j \in N, \mathcal{H}, g \simeq_{j}{ }^{\backslash(n, n+1)} \mathcal{H}, g a$ iff $\mathcal{H}^{\prime}, g \simeq_{j}{ }^{(n, n+1)} \mathcal{H}^{\prime}, g a$.

Proof. The proof is by induction on the length of the witness sequence for $n+1$-bisimulation closure reachable from $\mathcal{H}$. The base case is bisimilarity of identical structure. Now assume the claim holds for sequences of length $k$. Now, assume that $i$ is the witness agent and $\left(h e, h^{\prime} f\right)$ the witness pair to obtain $\mathcal{H}^{k+1}$ from $\mathcal{H}^{k}$. From the definition of the witness sequence we have $\mathcal{H}^{k}$, he $\simeq_{i}^{\backslash(n, n+1)} \mathcal{H}^{k}, h^{\prime} f$. By inspection of the clause of a $j$-bisimulation, we can see 
that for all $j \in N, \simeq_{j}{ }^{(n, n+1)}$-bisimilarity is invariant under $i$-linking $\simeq_{i}{ }^{\backslash(n, n+1)}$-bisimilar histories of length $n+1$ in a S5 ETL model. In particular we have for all $g, g a \in H$ that

$$
\begin{aligned}
\mathcal{H}^{k}, g \simeq_{j}{ }^{\backslash(n, n+1)} & \mathcal{H}^{k}, g a \\
& \text { iff } \mathcal{H}^{k+1}, g \simeq_{j}{ }^{(n, n+1)} \mathcal{H}^{k+1}, g a
\end{aligned}
$$

Moreover it is easy to see that by definition $\sim_{i}^{k+1}$ is still an equivalence relation and that $\sim_{i}^{k} \subseteq \sim_{i}^{k+1}$. Now by IH we have for all $g, g a \in H$ that $\mathcal{H}, g \simeq_{j} \backslash(n, n+1)$ $\mathcal{H}, g a$ iff $\mathcal{H}^{k}, g \simeq_{j}^{\backslash(n, n+1)} \mathcal{H}^{k+1}$, ga. Thus by (1) and transitivity of $\simeq_{j}{ }^{\backslash(n, n+1)}$-bisimilarity, and since $j$ was arbitrary the claim follows, concluding the induction step and the proof.

We can now prove our Main Theorem 16

Theorem 16. For an S5 epistemic-temporal ETL model, the following are equivalent:

1. $\mathcal{H}$ is isomorphic to asForest $(\mathcal{M}, \mathrm{P})$ for some epistemic model $\mathcal{M}$ and some state-dependent DEL protocol $\mathrm{P}$

2. $\mathcal{H}$ satisfies PR, NPTP, PT, SG, wUNM, and PS.

" $1 \Rightarrow 2 "$ : We fix an ETL model

$$
\mathcal{H}=\left\langle\Sigma, H,\left(\sim_{i}^{\mathcal{H}}\right)_{i \in N}, V\right\rangle=\operatorname{asForest}(\mathcal{M}, \mathrm{P}) .
$$

By definition of asForest, we know that $\operatorname{sForest}(\mathcal{M}, \mathrm{P})=\left\langle\Sigma, H,\left(\sim_{i}\right)_{i \in N}, V\right\rangle$ and $\sim_{i}^{\mathcal{H}}$ is the symmetric transitive closure of

$$
\begin{array}{r}
\sim_{i}^{0}=\sim_{i} \cup\{(w \sigma, w \sigma e) \in(H \times H) \mid \sigma e \in \mathrm{P}(w) \\
\text { and } \left.w \sigma \simeq_{i}^{\text {sForest }(\mathcal{M}, \mathrm{P})} w \sigma e\right\} .
\end{array}
$$

For any $h$ let $\mathcal{K}_{i}^{0}[h]=\left\{h^{\prime} \in H \mid h \sim_{i}^{0} h^{\prime}\right\}$. We start by proving that $\mathcal{H}$ has the given properties. Propositional Stability is immediate from our construction.

Perfect recall. Cf. Proposition 14 .

Synchronous grounding. Assume that $h \sim_{i}^{\mathcal{H}} h^{\prime}$ with $\operatorname{len}\left(h^{\prime}\right)>\operatorname{len}(h)$ for two histories $h$ and $h^{\prime}$ of the generated forest. If $h \leq h^{\prime}$, we are done. Otherwise, let $\sim_{i}^{0}$ denote the epistemic accessibility relation of the forest before closing off under transitivity, i.e., the relation given in (0). Since $h \sim_{i}^{\mathcal{H}} h^{\prime}$ and $\sim_{i}^{\mathcal{H}}$ is the transitive closure of $\sim_{i}^{0}$, we find a sequence of pairwise distinct histories $h_{1}, \ldots, h_{k}$ such that $h=h_{1} \sim_{i}^{0} \ldots \sim_{i}^{0} h_{k}=h^{\prime}$ and for all $1 \leq \ell<k$, either len $\left(h_{\ell}\right)=\operatorname{len}\left(h_{\ell+1}\right)$ or $h_{\ell+1}=h_{\ell} e_{\ell}$ for some $e_{\ell}$. Call the first kind "horizontal steps". By construction of the forest and the properties of the DEL product update, for any horizontal step $h_{\ell} \sim_{i}^{0} h_{\ell+1}$ there must also be epistemic accessibilities between all prefixes of $h_{\ell}$ and $h_{\ell+1}$ of equal length. This means that for each horizontal step $h_{\ell} \sim^{0} h_{\ell+1}$ between two histories of length greater than len $(h)$, we also get $h_{\ell}^{\prime} \sim^{0} h_{\ell+1}^{\prime}$ for the $h$-synchronous prefixes $h_{\ell}^{\prime}$ and $h_{\ell+1}^{\prime}$ of $h_{\ell}$ and $h_{\ell+1}$. This yields a $\sim_{i}^{0}$-path from $h$ to the $h$-synchronous prefix of $h^{\prime}$. Since $\sim_{i}^{\mathcal{H}}$ contains the transitive closure of $\sim_{i}^{0}$, we have shown the claim.

Perfect Tracking. We prove that $h \sim_{i}^{\mathcal{H}}$ he implies $h \simeq_{i}^{\mathcal{H} \backslash(\operatorname{len}(h), \operatorname{len}(h)+1)} h e$ for any $h$. Assume without loss of generality that len $(h)=n$ and that $h \sim_{i}^{\mathcal{H}} h e$. Now assume for contradiction that for all $h^{\prime}$ with len $\left(h^{\prime}\right)=n$ and $h \sim_{i}^{\mathcal{H}} h^{\prime}$ there is no event $f$ such that $h^{\prime} f \sim_{i}$ he and $h^{\prime} \simeq_{i}^{\mathcal{H} \backslash(n, n+1)} h^{\prime} f$. By construction, it means that none of the histories in $\mathcal{K}_{i}[h]$ with length $n$ were $i$-connected to a state in $\mathcal{K}_{i}[h e]$ by the bisimilarity condition. It follows that $\sim_{i}^{\mathcal{H}} \cap\left(\left(\mathcal{K}_{i}^{0}[h] \cap H_{n}\right) \times\right.$ $\left.\left(\mathcal{K}_{i}^{0}[h e] \cap H_{n+1}\right)\right)=\emptyset$, but this set is also empty after closure, i.e., $\sim_{i}^{\mathcal{H}} \cap\left(\left(\mathcal{K}_{i}[h] \cap H_{n}\right) \times\left(\mathcal{K}_{i}[h e] \cap H_{n+1}\right)\right)=\emptyset$ contradicting our hypothesis. Thus by reduction there must be some history $h^{\prime}$ with len $\left(h^{\prime}\right)=n$ and

$$
h \sim_{i}^{\mathcal{H}} h^{\prime}
$$

and some event $f$ such that

$$
h^{\prime} f \sim_{i}^{\mathcal{H}} h e
$$

and

$$
h^{\prime} \simeq_{i}^{\mathcal{H} \backslash(n, n+1)} h^{\prime} f .
$$

Since $\sim_{i}^{\mathcal{H}}$ is an equivalence relation it follows from (2), (3), (4) and transitivity of $i$-bisimilarity that $h \simeq_{i}^{\mathcal{H} \backslash(n, n+1)} h e$.

No pure time perception. Assume that $h \simeq_{i}^{\mathcal{H} \backslash(n, n+1)} h e$, then by the bisimilarity clause in the construction we have immediately $h \sim_{i}^{\mathcal{H}} h e$.

Weak Uniform no Miracles. Let $e=\left(\mathcal{E}, e_{1}\right)$ and $f=\left(\mathcal{E}^{\prime}, f_{1}\right)$. Assume that there are he and $h^{\prime} f$ with $\operatorname{len}(h)=\operatorname{len}\left(h^{\prime}\right), h e \sim_{i}^{\mathcal{H}} h^{\prime} f$ and $h \mathcal{\psi}_{i}^{\mathcal{H}} h e$. By Lemma 15 we have also $h \sim_{i}^{\mathcal{H}} h^{\prime}$. By construction we have S5-closure of the epistemic relations, if we had $h \sim_{i}^{\mathcal{H}}$ $g \sim_{i}^{\mathcal{H}} g a \sim_{i}^{\mathcal{H}} h e$, we would also have $h \sim_{i}^{\mathcal{H}} h e$, so $\sim_{i}^{\mathcal{H}} \cap\left(\left(\mathcal{K}_{i}[h] \cap H_{n}\right) \times\left(\mathcal{K}_{i}[h e] \cap H_{n+1}\right)\right)=\varnothing$. Thus $h e \sim_{i}^{\mathcal{H}} h^{\prime} f$ is not obtained by closure, but by product update and we have $\mathcal{E}=\mathcal{E}^{\prime}$. By construction and definition product update this also implies that

$$
e_{1} \sim_{i}^{\mathcal{E}} f_{1} .
$$

Now assume that $g, g^{\prime}, g e, g^{\prime} f \in H$ and

$$
g \sim_{i}^{\mathcal{H}} g^{\prime} .
$$


Now by (5), (6) and product update we have $g e \sim_{i}^{\mathcal{H}}$ $g^{\prime} f$.

This finishes the proof of the necessity of our conditions.

" $2 \Rightarrow 1$ ": Given some ETL model

$$
\mathcal{H}^{\mathrm{ETL}}=\left\langle\Sigma, H,\left(\sim_{i}^{\mathrm{ETL}}\right)_{i \in N}, V\right\rangle
$$

satisfying the stated conditions we show how to construct a matching initial epistemic model and a DEL protocol. We start with the definition of the initial epistemic model $\mathcal{M}_{0}=\left\langle W,\left(\sim_{i}^{\mathrm{DEL}}\right)_{i \in N}, \hat{V}\right\rangle$ :

- $W:=\{h \in H \mid \operatorname{len}(h)=1\}$.

- Set $h \sim_{i}^{\text {DEL }} h^{\prime}$ iff $h \sim_{i}^{\text {ETL }} h^{\prime}$.

- For every $p \in$ PROP, $\hat{V}(p)=V(p) \cap W$.

Now we construct the $j$ th event model $\mathcal{E}_{j}=$ $\left\langle E_{j},\left(\sim_{i}^{j}\right)_{i \in N}, \operatorname{pre}_{j}\right\rangle$ :

- $E_{j}:=\{e \in \Sigma \mid$ there is a history he $\in H$ with $\operatorname{len}(h)=j\}$.

- Set $a \sim_{i}^{j} b$ iff the following condition holds:

- there exists $h a, h^{\prime} b \in H$ such that $\operatorname{len}(h)=$ $\operatorname{len}\left(h^{\prime}\right)=j, h a \sim_{i}^{\mathrm{ETL}} h^{\prime} b$ and $h \mathcal{\psi}_{i}^{\mathrm{ETL}} h a$.

- Since we shall be using state-dependent protocols, we can set trivial preconditions $\operatorname{pre}_{j}(e)=\top$ and therefore shall not need a Bisimulation Invariance condition.

Finally we update the protocol for each state: if $w e_{1} \ldots e_{n} \in H$ we add $e_{1} \ldots e_{n}$ to $\mathrm{P}(w)$. We are now done with our construction. It gives rise to a forest

$$
\operatorname{asForest}\left(\mathcal{M}_{0}, \mathrm{P}\right):=\left(\Sigma, H,\left\{\sim_{i}^{\mathrm{DEL}}\right\}_{i \in N}, V\right),
$$

Claim 23. Let $\sim_{i}^{\mathrm{ETL}}$ be the epistemic relation in the given ETL model. Let $\sim_{i}^{\text {DEL }}$ be the epistemic relation in the forest induced over the just constructed epistemic model and DEL protocol. We have:

$$
h \sim_{i}^{\text {ETL }} h^{\prime} \text { iff } h \sim_{i}^{\text {DEL }} h^{\prime}
$$

Proof of Claim 23. The proof is by induction on the maximal length of histories we consider. Given that the claim holds for all pairs of histories in $H_{\leq n} \times H_{\leq n}$, our induction step works as follows: we prove a similarity claim for pairs in $H_{n+1} \times H_{n+1}$ (Part A), then we prove isomorphism for pairs of histories in $H_{j} \times H_{n+1}$ with $1 \leq j<n+1$ (Part B), and finally we prove isomorphism for pairs in $H_{n+1} \times H_{n+1}$ (Part C).
Base Case. Isomorphism for histories in $H_{\leq 1} \times H_{\leq 1}$ is immediate from the construction.

Induction step. Assume that we proved everything for histories in $H_{\leq n} \times H_{\leq n}(\mathrm{IH})$.

Part A: If he $\sim_{i}^{\mathrm{ETL}} h^{\prime} f$ then he $\sim_{i}^{\mathrm{DEL}} h^{\prime} f$ or $h, h^{\prime}, h e, h^{\prime} f$ are all $\simeq_{i}^{\mathcal{H}^{\mathrm{ETL}} \backslash(n, n+1)}$-bisimilar. If $h e \sim_{i}^{\text {DEL }} h^{\prime} f$ then $h e \sim_{i}^{\text {ETL }} h^{\prime} f$ or $h, h^{\prime}, h e, h^{\prime} f$ are all $\simeq_{i}^{\text {asForest }\left(\mathcal{M}_{0}, \mathrm{P}\right) \backslash(n, n+1)}$-bisimilar.

From ETL model to Generated DEL. Assume that $h e \sim_{i}^{\text {ETL }} h^{\prime} f$. By Lemma 15 it follows that $h \sim_{i}^{\text {ETL }} h^{\prime}$. Thus by IH

$$
h \sim_{i}^{\mathrm{DEL}} h^{\prime}
$$

Now we are in one of two cases.

Case 1: there are histories $g e, g^{\prime} f$ with $\operatorname{len}(g)=$ $\operatorname{len}\left(g^{\prime}\right)=n, g e \sim_{i}^{\mathrm{ETL}} g^{\prime} f$ and $g \mathcal{\chi}_{i}^{\mathrm{ETL}} g e$, and then by construction it follows that

$$
e \sim_{i}^{\text {DEL }} f
$$

And thus from (7) and (8) by product update $h e \sim_{i}^{\text {DEL }}$ $h^{\prime} f$

Case 2: for all histories $g, g^{\prime}$ with len $(g)=\operatorname{len}\left(g^{\prime}\right)=n$ and $g e \sim_{i}^{\text {ETL }} g^{\prime} f$ we have $g \sim_{i}^{\text {ETL }} g e$ and from Lemma 15 it follows that $g \sim_{i}^{\text {ETL }} g^{\prime}$ and by the fact that $\sim_{i}^{\text {ETL }}$ is an equivalence relation, that $g \sim_{i}^{\text {ETL }}$ $g^{\prime} f$ and by Perfect Tracking that these four histories are bisimilar and that successive in these four are $\simeq_{i}^{\mathcal{H}^{\mathrm{ETL}} \backslash(n, n+1)}$-bisimilar. This in particular true of $h, h^{\prime}, h e, h^{\prime} f$.

From Generated DEL to ETL model. Assume that

$$
h e \sim_{i}^{\text {DEL }} h^{\prime} f
$$

By Lemma 15 it follows that

$$
h \sim_{i}^{\text {DEL }} h^{\prime}
$$

We are in one of two cases.

Case 1: Either $e \sim_{i}^{\text {DEL }} f$ and thus by construction there exists some histories $g e$ and $g^{\prime} f$ such that

$$
\operatorname{len}(g)=\operatorname{len}\left(g^{\prime}\right)=n
$$

and

$$
g \mathcal{\chi}_{i}^{\mathrm{ETL}} g e \& g e \sim_{i}^{\mathrm{ETL}} g^{\prime} f
$$

But then by weak uniform no miracles it follows from (10), 111), 12 and the fact that len $(h)=\operatorname{len}\left(h^{\prime}\right)$ that $h e \sim_{i}^{\text {ETL }} h^{\prime} f$

Case 2: $e \chi_{i}^{\text {DEL }} f$ but then by construction and from (9) and 10 it follows that $h \sim_{i}^{\text {DEL }} h e, h \sim_{i}^{\text {DEL }}$ 
$h^{\prime} f$ and these four histories are $\simeq_{i}^{\operatorname{asForest}\left(\mathcal{M}_{0}, \mathrm{P}\right) \backslash(n, n+1)}$ bisimilar. In particular $h \simeq_{i}^{\text {asForest }\left(\mathcal{M}_{0}, \mathrm{P}\right) \backslash(n, n+1)} h e$ and $h^{\prime} \simeq_{i}^{\text {asForest }\left(\mathcal{M}_{0}, \mathrm{P}\right) \backslash(n, n+1)} h^{\prime} f$. Concluding this part of the induction step.

Part B: We prove isomorphism for histories in $H_{\leq n} \times$ $H_{n+1}$.

From ETL model to Generated DEL. Let $1 \leq j \leq n$. Assume $h \in H_{j}, h^{\prime} e \in H_{n+1}$ and $h \sim_{i}^{\text {ETL }} h^{\prime} e$. But by synchronous grounding there must be a prefix $h_{j}^{*} e_{j+1} \in H_{j+1}$ of $h^{\prime} e$ such that $h \sim_{i}^{\text {ETL }} h_{j}^{*}$. Since $h_{j}^{*} \sim_{i}^{\mathrm{ETL}} h \sim_{i}^{\mathrm{ETL}} h^{\prime} e$, with perfect recall it follows that

$$
\mathrm{EE}\left(h_{j}^{*}\right) \approx \mathrm{EE}\left(h^{\prime} e\right)
$$

Now assume for contradiction that a pair intermediate histories immediately succeeding each other between $h_{j}^{*}$ and $h^{\prime} e$ are not epistemically connected, then $\mathrm{EE}\left(h_{j}^{*}\right) \not \mathrm{EE}\left(h^{\prime} e\right)$, contradicting 13$)$. Thus by reduction all intermediate histories must therefore be epistemically connected, and in particular $h_{j}^{*} \sim_{i}^{\mathrm{ETL}} h_{j}^{*} e_{j+1}$ and $h_{j}^{*} e_{j+1} \sim_{i}^{\text {ETL }} h^{\prime} e$. If $\operatorname{len}\left(h_{j}^{*} e_{j+1}\right)<\operatorname{len}\left(h^{\prime} e\right)$ we can iterate the argument. In general we obtain a path of the form:

$$
\begin{gathered}
h \sim_{i}^{\mathrm{ETL}} h_{j}^{*} \sim_{i}^{\mathrm{ETL}} h_{j}^{*} e_{j+1} \sim_{i}^{\mathrm{ETL}} h_{j}^{*} e_{j+1} e_{j+2} \sim_{i}^{\mathrm{ETL}} \ldots \\
\sim_{i}^{\mathrm{ETL}} h_{j}^{*} e_{j+1} e_{j+2} \ldots e_{n-j}=h^{\prime} \sim_{i}^{\mathrm{ETL}} h^{\prime} e
\end{gathered}
$$

By the induction hypothesis, we know that

$$
\mathcal{H}^{\mathrm{ETL}} \mid \leq n \text { is isomorphic to asForest }\left(\mathcal{M}_{0}, \mathrm{P}\right) \mid \leq n
$$

From (14) we have in particular $h^{\prime} \sim_{i}^{\text {ETL }} h^{\prime} e$. It follows then from Perfect Tracking that $h^{\prime} \simeq_{i}^{\mathcal{H}^{\mathrm{ETL}} \backslash(n, n+1)}$ $h^{\prime}$ e. Moreover from Part A, Fact 22 and Observation 20 it follows that

$$
h^{\prime} \simeq_{i}^{\operatorname{asForest}\left(\mathcal{M}_{0}, \mathrm{P}\right) \backslash(n, n+1)} h^{\prime} e
$$

But then by construction we have

$$
h^{\prime} \sim_{i}^{\mathrm{DEL}} h^{\prime} e
$$

But from (14), 15) and (17) and the closure condition of the construction it follows that $h \sim_{i}^{\text {DEL }} h^{\prime} e$. Since $j$ was arbitrary, this concludes this direction of this part of the induction step.

From Generated DEL to ETL model. The first part of the argument is identical, giving us a path:

$$
\begin{aligned}
h \sim_{i}^{\mathrm{DEL}} & h_{j}^{*} \sim_{i}^{\mathrm{DEL}} h_{j}^{*} e_{j+1} \sim_{i}^{\mathrm{DEL}} \\
& h_{j}^{*} e_{j+1} e_{j+2} \sim_{i}^{\mathrm{DEL}} \ldots e_{n-j}=h^{\prime} \sim_{i}^{\mathrm{DEL}} h^{\prime} e
\end{aligned}
$$

$h^{\prime} \simeq_{i}^{\text {asForest }\left(\mathcal{M}_{0}, \mathrm{P}\right) \backslash(n, n+1)} h^{\prime} e$ follows from construction. From the same argument as in the other direction, it follows $h^{\prime} \simeq_{i}^{\mathcal{H}^{\mathrm{ETL}} \backslash(n, n+1)} h^{\prime} e$. Now it follows from no pure time perception that

$$
h^{\prime} \sim_{i}^{\mathrm{ETL}} h^{\prime} e
$$

By $I H$ we know that the $\mathcal{H}^{\mathrm{ETL}} \mid \leq n$ is isomorphic to

$$
\operatorname{asForest}\left(\mathcal{M}_{0}, \mathrm{P}\right) \mid \leq n
$$

From 18), 20], 19) and the fact that $\sim_{i}^{\text {ETL }}$ is an equivalence relation, we have $h \sim_{i}^{\mathrm{ETL}} h^{\prime} e$. Again $j$ was arbitrary, concluding this direction and this part of the induction step.

Part $C$ : We prove isomorphism for histories in $H_{n+1} \times$ $H_{n+1}$.

From ETL model to Generated DEL. Assume for that he $\sim_{i}^{\mathrm{ETL}} h^{\prime} f$. Now assume for contradiction that he $\chi_{i}^{i}$ EL $h^{\prime} f$. Then by Part $\mathrm{A}$ we have that $h, h^{\prime}, h e, h^{\prime} f$ are all $\simeq_{i}^{\mathcal{H}^{\mathrm{ETL}} \backslash(n, n+1)}$-bisimilar. Moreover by our usual argument we have $h \sim_{i}^{\text {ETL }} h^{\prime}$. By induction hypothesis and Part B this is also the case in the DEL generated forest and by construction and closure we have $h e \sim_{i}^{\text {DEL }} h^{\prime} f$. Contradiction. Thus by reduction we have indeed $h e \sim_{i}^{\text {DEL }} h^{\prime} f$.

From Generated DEL to ETL model.

Assume for that $h e \sim_{i}^{\text {DEL }} h^{\prime} f$. Now assume for contradiction that he $\chi_{i}^{\text {ETL }} h^{\prime} f$. Then by Part A we have that $h, h^{\prime}, h e, h^{\prime} f$ are all $\simeq_{i}^{\text {asForest }\left(\mathcal{M}_{0}, \mathrm{P}\right) \backslash(n, n+1)}$ bisimilar. Moreover by our usual argument we have $h \sim_{i}^{\text {DEL }} h^{\prime}$ and by construction $h \sim_{i}^{\text {DEL }} h e$ and $h^{\prime} \sim_{i}^{\text {DEL }} h^{\prime} f$. By induction hypothesis and Part B this is also the case in the ETL model. Since this is an S5 ETL model we have he $\sim_{i}^{\mathrm{ETL}} h^{\prime} f$. Contradiction. Thus by reduction we have indeed $h e \sim_{i}^{\mathrm{ETL}} h^{\prime} f$.

This concludes this part of the induction step, the induction step, this direction of the proof and the proof of the claim.

The sufficiency of our conditions follows from Claim 24.

\section{B Proof of Theorem 19}

" $1 \Rightarrow 2$ ": We start by proving that the epistemic temporal forests generated from an initial model, a clock tick function and a protocol according to the procedure given in definition have the given properties. By examination of the proofs given in the preceding representation theorem, it is clear that the preceding properties (except for NPTP) are still true of any model generated with a clock-ticking function even if one does not add some epistemic link between two immediately 
succeeding bisimilar histories. Below we give, for the interesting cases, the intuition why properties (except for NPTP) are preserved).

Perfect recall. The main idea of the soundness argument (Proposition 14) was to prove inductively that merging information sets of bisimilar successors (adding 'vertical' links) preserves perfect recall. Forests generated according to clock-tick functions are intuitively intermediate structures between synchronously DEL generated forests and their asynchronously generated cousins.

Synchronous grounding. 'Horizontal uncertainties' still originate by product update and product update is still applied to synchronous slices.

Perfect Tracking. Clock tick functions allow agents to distinguish epistemically equivalent states. So it intuitively speaking it allow them to distinguish strictly more states than in the asynchronous construction, so perfect tracking is preserved (while NPTP will general not hold any longer).

Weak Uniform no Miracles. The case of weak uniform no miracles is interesting. The soundness under clock tick functions stems from the fact that under Perfect Recall, Synchronous Grounding and S5, the generated forests will always be isomorphic to one that could be generated by a well-behaved clock tick function: well-behaved in the sense that it fulfills a form closure: if $w h(\mathcal{E}, e) \sim_{i} w^{\prime} h^{\prime}(\mathcal{E}, f)$ and $i \in c(w, h,(\mathcal{E}, e))$ then $i \in c\left(w^{\prime}, h^{\prime},(\mathcal{E}, f)\right)$.

This finishes the proof of the necessity of our conditions.

" $2 \Rightarrow 1 "$ : Given some ETL model satisfying the stated conditions we show how to construct a matching initial epistemic model, state-dependent DEL protocol and clock-ticking function. We start with the definition of the initial epistemic model $\mathcal{M}_{0}=\left\langle W,\left(\sim_{i}^{\text {DEL }}\right)_{i \in N}, \hat{V}\right\rangle$ :

- $W:=\{h \in H \mid \operatorname{len}(h)=1\}$.

- Set $h \sim_{i}^{\text {DEL }} h^{\prime}$ iff $h \sim_{i}^{\text {ETL }} h^{\prime}$.

- For every $p \in \operatorname{PrOP}, \hat{V}(p)=V(p) \cap W$.

Now we construct the $j$ th event model $\epsilon_{j}=$ $\left\langle E_{j},\left(\sim_{i}^{j}\right)_{i \in N}, \operatorname{pre}_{j}\right\rangle$ :

- $E_{j}:=\{e \in \Sigma \mid$ there is a history he $\in H$ with $\operatorname{len}(h)=j\}$.

- Set $a \sim_{i}^{j} b$ iff there are $h a, h^{\prime} b \in H$ such that $\operatorname{len}(h)=\operatorname{len}(h)=j$ and $h a \sim_{i}^{\mathrm{ETL}} h^{\prime} b$.
- Since we shall be using state-dependent protocols, we can set trivial preconditions $\operatorname{pre}_{j}(e)=\top$ and therefore shall not need a Bisimulation Invariance condition.

We update the protocol for each state: if $w e_{1} \ldots e_{n} \in$ $H$ we add $e_{1} \ldots e_{n}$ to $\mathrm{P}(w)$. Finally for each triple $(h, \sigma, h \sigma e) \in H_{1} \times H_{j} \times H_{j+1}$ our clock ticking function is defined as $i \in \mathrm{c}\left(h, \sigma,\left(E_{j}, e\right)\right)$ iff $h \sigma \chi_{i}^{\mathrm{ETL}} h \sigma e$.

Claim 24. Let $\sim_{i}^{\text {ETL }}$ be the epistemic relation in the given ETL model. Let $\sim_{i}^{\text {DEL }}$ be the epistemic relation in the forest induced over the just constructed epistemic model and DEL protocol. We have:

$$
h \sim_{i}^{\text {ETL }} h^{\prime} \text { iff } h \sim_{i}^{\text {DEL }} h^{\prime}
$$

Proof of Claim 24. The proof follows closely the strategy of the previous theorem, i.e., is by induction on the maximal length of histories we consider. Given that the claim holds for all pairs of histories in $H_{\leq n} \times H_{\leq n}$, we prove a similarity claim for pairs in $H_{n+1} \times H_{n+1}$ (Part A), then we prove isomorphism for pairs of histories in $H_{j} \times H_{n+1}$ with $1 \leq j<n+1$ (Part B), and finally we prove isomorphism for pairs in $H_{n+1} \times H_{n+1}$ (Part C). To keep this appendix focused on the important changes we try not to repeat arguments when they are identical in the previous proof.

The base case for histories in $H_{\leq 1} \times H_{\leq 1}$ and the proof of part the part A of the induction step follows the same argument. However we shall prove a stronger claim (that was not necessary in the proof of the previous theorem):

Part A:

1. If $h e \sim_{i}^{\mathrm{ETL}} h^{\prime} f$ then $h e \sim_{i}^{\mathrm{DEL}} h^{\prime} f$ or we have both that $h, h^{\prime}, h e, h^{\prime} f$ are all $\simeq_{i}^{\mathcal{H}^{\mathrm{ETL}} \backslash(n, n+1)}$-bisimilar and that they are all $\sim_{i}^{\text {ETL }}$-connected.

2. If he $\sim_{i}^{\mathrm{DEL}} h^{\prime} f$ then $h e \underset{i}{\sim \mathrm{ETL}} h^{\prime} f$ or $h, h^{\prime}, h e, h^{\prime} f$ are all $\simeq_{i}^{\operatorname{clickForest}\left(\mathcal{M}_{0}, \mathrm{P}\right) \backslash(n, n+1)}$ - $_{\text {- }}$ bisimilar and moreover $h \sim_{i}^{\text {DEL }} h e$ and $h^{\prime} \sim_{i}^{\text {DEL }}$ $h^{\prime} f$.

Part B: We prove isomorphism for histories in $H_{\leq n} \times$ $H_{n+1}$.

From ETL model to Generated DEL. From the same argument as in the proof of the previous theorem, we obtain a path of the form:

$$
\begin{gathered}
h \sim_{i}^{\mathrm{ETL}} h_{j}^{*} \sim_{i}^{\mathrm{ETL}} h_{j}^{*} e_{j+1} \sim_{i}^{\mathrm{ETL}} h_{j}^{*} e_{j+1} e_{j+2} \sim_{i}^{\mathrm{ETL}} \ldots \\
\sim_{i}^{\mathrm{ETL}} h_{j}^{*} e_{j+1} e_{j+2} \ldots e_{n-j}=h^{\prime} \sim_{i}^{\mathrm{ETL}} h^{\prime} e .
\end{gathered}
$$


By the induction hypothesis, we know that

$$
\mathcal{H}^{\mathrm{ETL}} \mid \leq n \text { is isomorphic to } \operatorname{clickForest}\left(\mathcal{M}_{0}, \mathrm{P}\right) \mid \leq n
$$

From 21 we have in particular

$$
h^{\prime} \sim_{i}^{\mathrm{ETL}} h^{\prime} e
$$

It follows then from Perfect Tracking that $h^{\prime} \simeq_{i}^{\mathcal{H}^{\mathrm{ETL}} \backslash(n, n+1)} h^{\prime}$ e. Moreover from Part A, Fact 22 and Observation 20 it follows that

$$
h^{\prime} \simeq_{i}^{\operatorname{clickForest}\left(\mathcal{M}_{0}, \mathrm{P}\right) \backslash(n, n+1)} h^{\prime} e
$$

Let $\sigma$ be the sequence such that $h^{\prime}=h^{\prime}[1] \sigma$. It follows from (23) by construction of the clock ticking function that

$$
i \notin \mathrm{c}\left(h^{\prime}[1], \sigma,\left(E_{n+1}, e\right)\right)
$$

But then by construction we have

$$
h^{\prime} \sim_{i}^{\mathrm{DEL}} h^{\prime} e
$$

But from (21), 22) and (26) and the closure condition of the construction it follows that $h \sim_{i}^{\text {DEL }} h^{\prime} e$. Since $j$ was arbitrary, this concludes this direction of this part of the induction step.

From Generated DEL to ETL model. The first part of the argument is identical, giving us a path:

$$
\begin{aligned}
h \sim_{i}^{\mathrm{DEL}} & h_{j}^{*} \sim_{i}^{\mathrm{DEL}} h_{j}^{*} e_{j+1} \sim_{i}^{\mathrm{DEL}} \\
& h_{j}^{*} e_{j+1} e_{j+2} \sim_{i}^{\mathrm{DEL}} \ldots e_{n-j}=h^{\prime} \sim_{i}^{\mathrm{DEL}} h^{\prime} e
\end{aligned}
$$

Let $\sigma$ be the sequence such that $h^{\prime}=h^{\prime}[1] \sigma$. It follows by construction that

$$
i \notin \mathrm{c}\left(h^{\prime}[1], \sigma,\left(E_{n+1}, e\right)\right)
$$

But then by construction of the clock tick function it follows that

$$
h^{\prime} \sim_{i}^{\mathrm{ETL}} h^{\prime} e
$$

By $I H$ we know that the $\mathcal{H}^{\mathrm{ETL}} \mid \leq n$ is isomorphic to

$$
\operatorname{clickForest}\left(\mathcal{M}_{0}, \mathrm{P}\right) \mid \leq n
$$

From 27), 30, 29 and the fact that $\sim_{i}^{\mathrm{ETL}}$ is an equivalence relation, we have $h \sim_{i}^{\mathrm{ETL}} h^{\prime} e$. Again $j$ was arbitrary, concluding this direction and this part of the induction step.

Part $C$ : We prove isomorphism for histories in $H_{n+1} \times$ $H_{n+1}$.

From ETL model to Generated DEL. Assume for that he $\sim_{i}^{\mathrm{ETL}} h^{\prime} f$. Now assume for contradiction that he $\chi_{i}^{\text {DEL }} h^{\prime} f$. Then by Part $\mathrm{A}$ we have that $h, h^{\prime}, h e, h^{\prime} f$ are all $\sim_{i}^{\mathrm{ETL}}$-connected. By induction hypothesis and Part B this is also the case in the DEL generated forest. By closure we have he $\sim_{i}^{\text {DEL }} h^{\prime} f$. Contradiction. Thus by reduction we have indeed $h e \sim_{i}^{\mathrm{DEL}} h^{\prime} f$.

From Generated DEL to ETL model.

Assume for that $h e \sim_{i}^{\text {DEL }} h^{\prime} f$. By our usual argument we have $h \sim_{i}^{\text {DEL }} h^{\prime}$. Now assume for contradiction that

$$
h e \chi_{i}^{\mathrm{ETL}} h^{\prime} f
$$

By Part B and (31) we have $h \sim_{i}^{\text {DEL }} h e$ and $h^{\prime} \sim_{i}^{\text {DEL }}$ $h^{\prime} f$. By induction hypothesis and Part B this is also the case in the ETL model. Since this is an S5 ETL model we have he $\sim_{i}^{\text {ETL }} h^{\prime} f$. Contradiction. Thus by reduction we have indeed he $\sim_{i}^{\mathrm{ETL}} h^{\prime} f$.

The sufficiency of our conditions follows from Claim 24. 